\title{
Ripening classification of bananas (Musa acuminate) using electrical impedance spectroscopy and support vector machine
}

\begin{abstract}
Bananas are one of the most nutritious and widely available food products in the world. An important step of the mass production of bananas (Musa acuminate) is determining it's ripening stages, indicating that it is ready for consumption, and with the most concentration of important nutrients, what will not be achieved in under-ripening or over-ripening. The use of faster and easier assays to determinate the optimum ripening stage will allow the production of fruits with quality and consistence, increasing also the producer income. The industry standard for this process relies on the use of the Von Loesecke scale through visual analysis of the fruits which can result in significant discrepancies and commercial losses. Biochemical analyses can also be used but are time-consuming. The objective here is to investigate and develop a ripening classification test for bananas by using electrical impedance spectroscopy together with a support vector machine algorithm. 100 samples of bananas at 3 ripening stages were used for measuring both impedance modulus and phase of the impedance in the frequency range from $50 \mathrm{~Hz}$ to $1 \mathrm{MHz}$. Results showed that this process is able to identify ripeness stages with $100 \%$ accuracy with low requirements for processing time. The techniques presented here might be applied in large scale production in order to make the automation process of the harvest time more reliable and profitable.
\end{abstract}

Volume 6 Issue 4 - 2020

\author{
William Laus Bertemes,' Ricardo Cavalieri," \\ Aline Torres Paré,' Julia Spessatto,' Daiani \\ Savi,' Pedro Bertemes-Filho² \\ 'Department of Biomedical Sciences, Catholic University \\ Center of Santa Catarina, Brazil \\ ${ }^{2}$ Department of Electrical Engineering, State University of Santa \\ Catarina, Brazil
}

\begin{abstract}
Correspondence: Pedro Bertemes-Filho, Department of Electrical Engineering, State University of Santa Catarina, Joinville, Brazil, Email pedro.bertemes@udesc.br
\end{abstract}

Received: October 30, 2020 | Published: November 10, 2020

Keywords: electrical impedance spectroscopy, biological tissue, ripening classification, support vector machine

Abbreviations: CSV, comma-separated value; EIS, electrical impedance spectroscopy; SVM, support vector machines

\section{Introduction}

Banana (Musa acuminate) is considered one of the most consumed fruits in the world because of its rich nutritional value and its flavor. For these reasons, banana production is one of the biggest and most lucrative fruit in many countries. Considering the weather sensitive nature of the product, the determination of ripening stage is crucial, ensuring the quality of the product and preventing postharvest losses. ${ }^{1}$ Generally, a climacteric fruit has a high respiratory rate and a high ethylene production in the beginning of the postharvest ripening process, which loss of rigidity and the presence of signs of enzymatic browning while starch is transformed into simple carbohydrates. ${ }^{2,3}$ Most biochemical methods of assessing the ripening process are limited by processing times and the requirement for expensive laboratory equipment and destruction of the fruit. ${ }^{4}$ To avoid losses in the determination of the ripening quality of banana fruits during the ripening treatment, a non-destructive detecting method are required. ${ }^{5-7}$ The ideal time of harvest usually takes place during the initial ripening stages, while the fruit still displays a green coloration. The first observable sign of ripening is a color change from green to yellow, that are described based on Von Loesecke scale classification, ${ }^{8}$ which describes the visual properties of each stage in the post-harvest ripening process. Therefore, the use of only human visual inspection can result in significant discrepancies, labour-intensive process, interpersonal variation in the analysis and commercial losses.

Optoelectronic methods may be of great use for improving such a visual inspection with a quite high sensitivity. The capture of images and theirs following data process has been widely used for many types of inspection, including face recognition and food quality detection. The imaging method requires many measured data and it is time consuming and demands a quite complex hardware approach. Characterization of biological samples with electrical stimulus has also been used over the last decades, as for example, the Electrical Impedance Spectroscopy (EIS). EIS is a method for determining the electrical response of a given structure (biological or otherwise) when a multifrequency alternate current is applied to it. Through the measurement of the resulting voltage across the sample, the structural composition of the material can be described in terms of a complex electrical impedance. ${ }^{9}$ This method has been used in many different applications, ranging from medical diagnosis to purely industrial applications with the aim to accurately describe both organic and inorganic materials. ${ }^{10}$ Considering the food industry, EIS has been used in the characterization and quality control of plant-based samples. ${ }^{11,12}$ This is possible because the electrical parameters extracted from EIS analysis can be direct indicators of the physiological and structural makeup of the biological tissue sampled. This ensures that biological processes that happen within the analyzed tissue can be detected and investigated by changing the characteristics of the signal which is injected into the sample during Impedance Spectroscopy..$^{13}$ One particular study describes the electrical spectrum change observed over time in the ripening process of a banana, ${ }^{14}$ validating the use of EIS as a more objective and data-driven alternative to determining the ripening stages of a fruit. However, this study did not implement any type of classification for the ripening stages of bananas. The growing trend in the use of artificial intelligence has been proven time and again in the interpretation of many different types of data. One of the most common classification algorithms used currently is called a Support Vector Machine (SVM) classifier, which is able to successfully classify high-dimensionality data with accuracy and low 
processing requirements. ${ }^{15} \mathrm{SVM}$ may be considered a more suitable method of classification once it can more reliably work within limited sample sizes and non-linear data. Based on this, the main objective of this work is to investigate and develop a ripening classification test for bananas (Musa acuminate) by using electrical impedance spectroscopy together with a support vector machine algorithm.

\section{Material and methods}

Data was extracted from 30 bananas (Musa acuminate) obtained from local producers (Santa Catarina, Brazil). Ripeness stage was established using the Von Loesecke scale by visual analysis. Nine banana samples were classified as Stage 1 (exemplified in Figure 1A), 11 samples at Stage 4 (Figure 1B) and 10 samples at Stage 6 (Figure 1C). After visual analysis, EIS data was collected from each sample utilizing 4 conductive needles ( $15 \mathrm{~mm}$ long), separated by $5 \mathrm{~mm}$ each other in a 4-pole configuration by inserting it into the sampled tissue. A sinusoidal voltage of $10 \mathrm{Vp}$ over a frequency from $50 \mathrm{~Hz}$ to $1 \mathrm{MHz}$ (100 points logarithmically spaced) was used to excite the samples and the current was measured by the HF12S Impedance Spectroscope manufactured by Zurich Instruments. Data is exported to a personal computer to a CSV file containing the impedance modulus and phase angle, resulting in a 200 measured points for each sample. Data preprocessing is performed using the Pandas Python library ${ }^{16}$ consisting of data normalization without the use of dimensionality reduction. From the 30 samples obtained, 16 were separated for the SVM training process and 14 as a testing pool for the algorithm. The SVM classifier was implemented with the use of the Sci-Kit Learn Python library in both linear and polynomial (to the third degree) kernels.

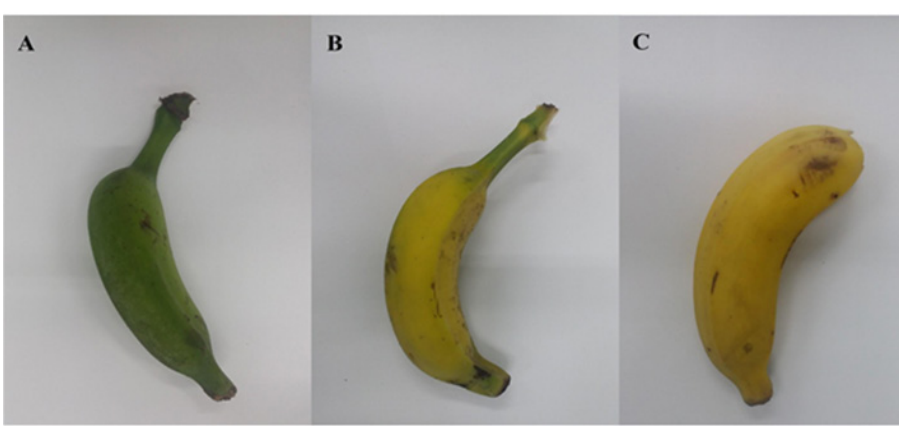

Figure I Ripening stages of the banana samples. (A) Stage I. (B) Stage 4. (C) Stage 6.

\section{Results and discussion}

Impedance data collected from samples shows clearly that different ripening stages are reflected in both absolute impedance (Figure 2A) and phase angle (Figure 2B) all along the frequency range. It is possible to infer from graphical analysis of the data obtained that the ripening stages are clearly differentiable from each other in terms of their electrical impedance. Figure 2 represents 5 randomly selected samples obtained from the measurements. A clear difference in the impedance modulus can be observed, especially in lower frequencies. It can be inferred that a computational intelligence algorithm would be able to separate each Stage, since there is a clear distinction between the curves obtained. Both linear and polynomial SVM classifier is trained on the gathered data and results are shown in Figure 3. Linear SVM performance achieves an accuracy of $100 \%$ on the determined test set, while the polynomial SVM achieves $92.85 \%$. Misclassification by the polynomial SVM occurs as a mix up between stages 2 and 3 and is likely due to edge cases. The confusion matrix, which is represented by Figure 3, confirm that the polynomial Kernel classifier is less accurate compared to the linear Kernel one, whether using normalization or not.
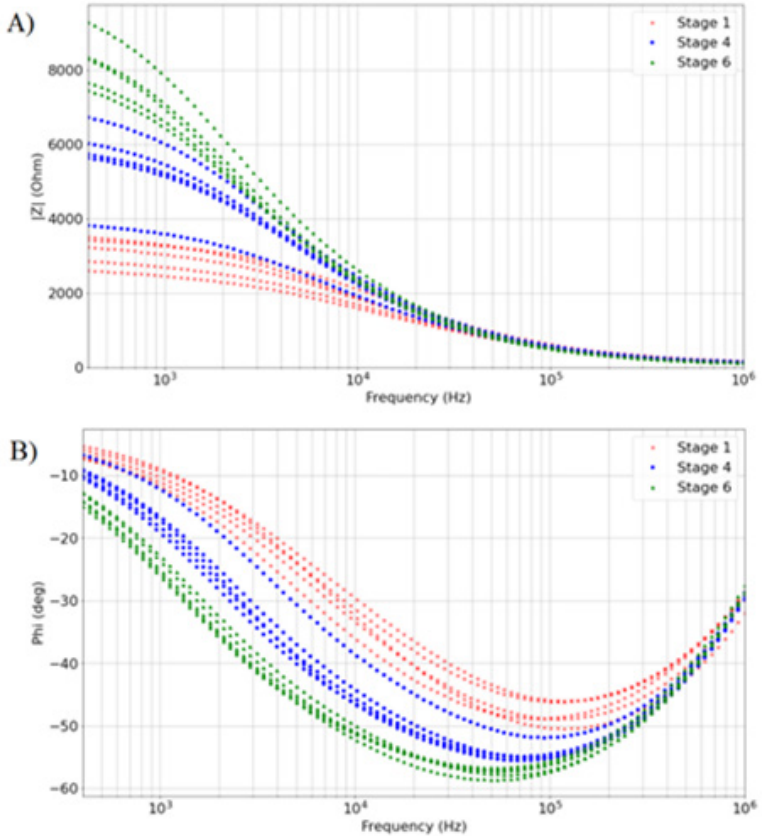

Figure 2 Spectrum of the impedance as a function of frequency.A) Modulus (l Z l). B) Phase angle (degree).
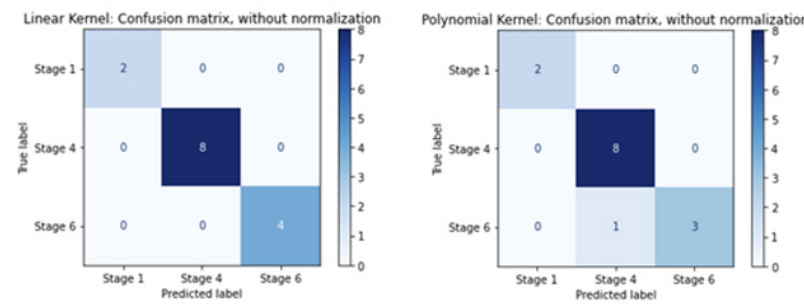

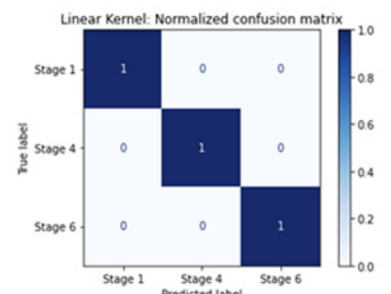

(A)

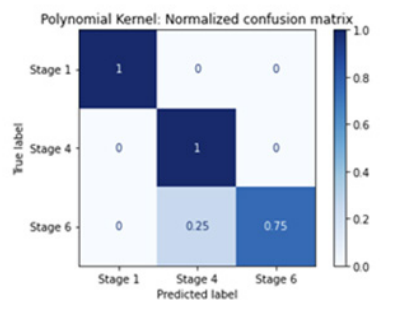

(B)
Figure 3 Confusion matrix comparing predicted value to true ones by SVM classifiers. (A) Linear kernel. (B) Polynomial kernel.

\section{Discussion}

Fruit ripening is a process where several physical-chemical and biological changes culminate in the desirable organoleptic characteristics, such as texture, color, odor, flavor and other properties that encourage its consumption. ${ }^{2}$ The determination of fruit ripening is a great obstacle to banana harvest, which will impact on better food quality with required nutrition level and commercial prices. ${ }^{14}$ So, the present study was carried out with the objective of classifying the ripening stages with less subjectivity, when compared with the visual scale of Von Loesecke, and of proposing a more robust methodology, by measuring the spectra in different bananas to consider the variations 
between different fruit specimens. From EIS data, it can be observed that this technique is quite a useful tool for indicating variations due to biochemical processes at different stages of banana ripening. The measurements show that the impedance at frequencies below $10 \mathrm{kHz}$ undergoes a gradual increase in each stage of ripening, from the least ripe to the ripest fruit (stages 1, 4 and 6 in Figure 2A). The low frequency impedance is not dependent of the intracellular resistivity, due to the cell membrane acting as a capacitor. ${ }^{17}$ Due to the fact that the vegetable tissue permittivity is a function of the movement of extracellular ions along the insulating membranes, ${ }^{9}$ it suggests that the increased humidity, total soluble solids (sugars) and decreased organic acids during the ripening process interfere the flow of electrical charges. As a result of this trend, there is an increase of impedance during this process. It can also be observed that there is a difference in the phase angle for frequencies below $100 \mathrm{kHz}$, increasing its magnitude from the ripest to the least ripe fruit (stages 6,4 , and 1 in Figure $2 \mathrm{~A}$ ). It is important to mention that the frequency range of the impedance does not be necessarily very wide, as it was observed in this study. A frequency range of 1 to $100 \mathrm{kHz}$ should be suitable for this type of classification, as it was not measured any difference in the impedance modulus for frequencies higher than $100 \mathrm{kHz}$ (Figure 2A). Care should be taken when comparing these results to other types of fruits due to theirs unique physical-chemical properties, which can be less sensitive to the measuring current for the EIS case. Nevertheless, by applying a higher voltage to the samples then a higher current is measure, increasing significantly the sensitivity of the EIS technique. The confusion matrices observed in Figure 3 are used to identify the situation where the polynomial kernel fails to classify correctly the maturation stage of the samples. This is done in order to evaluate in which situations the algorithm is most likely to mix up the results and serves to establish the linear kernel as the preferable alternative to this problem. As observed in Figure 3-B, out of the 4 samples labeled as stage 6 in the Von Loesecke maturation scale, only 3 were correctly identified by the polynomial kernel algorithm and one of them was misclassified as stage 4 (i.e., $75 \%$ accuracy in this classification). This error was not observed using the linear kernel algorithm, which was able to differentiate between stages 4 and 6 with $100 \%$ of accuracy for the test set. Overall, these results indicate that the change in physicalchemical properties during the ripening process of bananas seems to be quite sensitive to its ripening color, leading the visual inspection to uncertainties and under misclassification.

\section{Conclusion}

This study showed that EIS is a reliable method as an indicator of the ripening of bananas when used together with the SVM classifying algorithm. Both techniques identified the ripening banana stages as represented by the Von Loesecke scale, showing an accuracy of $100 \%$. We believe that this is a cost-effective alternative system to the subjective visual analysis of fruits with the use of EIS data and an artificial intelligence classifier. The techniques presented here might be applied in large scale production in order to make the automation process of the harvest time more reliable and profitable. Future experiments will be performed by comparing the banana ripeness with its Brix Degree, which is the sugar content of an aqueous solution where the fruit is dissolved, as well as measuring different varieties samples of bananas.

\section{Acknowledgments}

We thank the Catholic University Center of Santa Catarina for the institutional support and the State University of Santa Catarina for the financial support and for providing the infrastructure for carrying out this research.

\section{Conflicts of interest}

The authors declare that there are no conflicts of interest.

\section{References}

1. Almeida Clóvis Oliveira de. Produção e Problemas na Comercialização Embrapa. 2002:245-25.

2. Lima JD, Mendonça, JCDE. Características da fruta. Banana: Póscolheita. Brasilia: Embrapa Informação Tecnológica. 2001:80-85.

3. Maduwanthi SDT, Marapana R. Biochemical changes during ripening of banana : A review. 2017;2(5):AS166-169.

4. Islam Monzurul, Wahid Khan, Dinh Anh. Assessment of Ripening Degree of Avocado by Electrical Impedance Spectroscopy and Support Vector Machine. Journal of Food Quality. 2018.

5. CEAGESP. Normas de classificação de Banana. Programa Brasileiro para a modernização da horticultura e produção integrada de frutas. 2006;29(7)

6. Sarmento CAR. Determinação do ponto de colheita e avaliação da póscolheita de banana princesa utilizando biofilme. 2012:74.

7. Loesecke VHW. Bananas - Chemistry, physiology, technology. Interscience Publishers; 1950.

8. Bora GC, Donqing Lin, Pritha Bhattacharya, et al. Application of Bio-Image Analysis for Classification of Different Ripening Stages of Banana. Journal of Agricultural Science. 2015;7(2).

9. Bertemes-Filho P, Simini F. Bioimpedance in Biomedical Applications and Research. 2018

10. Dean DA, Ramanathan T, Machado D, et al. Electrical Impedance Spectroscopy Study of Biological Tissues. Journal of Electrostatics. 2008;23(1):1-7.

11. El Khaled D, Castellano NN, Gazquez JA, et al. Cleaner quality control system using bioimpedance methods: a review for fruits and vegetables. Journal of Cleaner Production. 2017;140:1749-1762.

12. Pliquett U. Bioimpedance: A review for food processing. Food Engineering Reviews. 2010;2(2): 74-94.

13. LIU, X. Electrical Impedance Spectroscopy Applied in Plant Physiology Studies. RMIT University; 2006

14. Chowdhury A, Tushar Kanti Bera, Dibyendu Ghoshal, et al. Electrical Impedance Variations in Banana Ripening: An Analytical Study with Electrical Impedance Spectroscopy. Journal of Food Process Engineering. 2017;40(2).

15. Vapnik V, Cortes C. Support-Vector Networks. Mach Learn. 1995;20:273-297.

16. Cavalieri R, Bertemes-Filho P. Plant tissue differentiation using electrical impedance spectroscopy with deep neural networks. International Journal of Biosensors \& Bioelectronics. 2020;6(1):20-23.

17. Martinsen OG, Grimnes S. Bioimpedance and Bioelectricity Basics. Academic press; 2015. 